\title{
Determination of Optimum Processing Temperature for Transformation of Glyceryl Monostearate ${ }^{1)}$
}

\author{
Toshio YaJima, ${ }^{* a}$ Shigeru ITAI, ${ }^{a}$ Hirofumi TAKeUchi, ${ }^{b}$ and Yoshiaki KaWAShima ${ }^{b}$ \\ ${ }^{a}$ Pharmaceutics Laboratory, Medicinal Research Laboratories, Taisho Pharmaceutical Co., Ltd.; 1-403 Yoshino-cho, \\ Saitama, Saitama 330-8530, Japan: and ${ }^{b}$ Gifu Pharmaceutical University; 5-6-1 Mitahora-Higashi, Gifu 502-8585, \\ Japan. Received April 8, 2002; accepted August 10, 2002
}

The purpose of this study was to clarify the mechanism of transformation from $\alpha$-form to $\beta$-form via $\beta^{\prime}$ form of glyceryl monostearate (GM) and to determine the optimum conditions of heat-treatment for physically stabilizing GM in a pharmaceutical formulation. Thermal analysis repeated twice using a differential scanning calorimeter (DSC) were performed on mixtures of two crystal forms. In the first run (enthalpy of melting: $\Delta H_{1}$ ), two endothermic peaks of $\alpha$-form and $\beta$-form were observed. However, in the second run (enthalpy of melting: $\Delta \mathrm{H}_{2}$ ), only the endothermic peak of the $\alpha$-form was observed. From a strong correlation observed between the $\beta$ form content in the mixture of $\alpha$-form and $\beta$-form and the enthalpy change, $\left(\Delta H_{1}-\Delta H_{2}\right) / \Delta H_{2}, \beta$-form content was expressed as a function of the enthalpy change. Using this relation, the stable $\beta$-form content during the heat-treatment could be determined, and the maximum $\beta$-form content was obtained when the heat-treatment was carried out at $50^{\circ} \mathrm{C}$. An inflection point existed in the time course of transformation of $\alpha$-form to $\beta$-form. It was assumed that almost all of $\alpha$-form transformed to $\beta^{\prime}$-form at this point, and that subsequently only transformation from $\beta^{\prime}$-form to $\beta$-form occurred. Based on this aspect, the transformation rate equations were derived as consecutive reaction. Experimental data coincided well with the theoretical curve. In conclusion, GM was transformed in the consecutive reaction, and $50^{\circ} \mathrm{C}$ was the optimum heat-treatment temperature for transforming GM from the $\alpha$-form to the stable $\beta$-form. ratio

Key words glyceryl monostearate; transformation; differential scanning calorimeter; polymorphism; enthalpy; transformation

Glyceryl monostearate (GM), a mixture of glyceryl esters of fatty acids, is composed of glyceryl monostearate $(65 \%)$, glyceryl monopalmitate $(30 \%)$ and glyceryl monomyristate $(5 \%)$. It has been widely used as an emulsifying agent in food preparations.

Maruyama et al. carried out precise studies of the polymorphism of $\mathrm{GM},{ }^{2,3)}$ and reported that four crystal forms of GM exist. Rapid cooling of melted GM yields the $\alpha$-form, which is successively transformed to the $\beta$-form via the $\beta^{\prime}$ form under ambient conditions. Since the $\alpha$-form is dispersible and foamy, it is often used as an emulsifying agent, foaming agent and preservative for bread, ice cream, cookies, etc. ${ }^{4)}$ On the other hand, the $\beta$-form of GM having higher melting point, higher density, is poorer in foaming and wetting than the $\alpha$-form.

We have applied $\beta$-form of GM to the pediatric formulation of clarithromycin (CAM) and succeeded in masking the bitter taste. ${ }^{5)}$ Our formulation was comprised of a wax matrix agglomerated using a spray-congealing technique. ${ }^{6}$ In the manufacturing process, GM, one of the components of the wax matrix, is transformed from the $\beta$-form to the $\alpha$-form and gradually transformed back to the $\beta$-form at ambient temperature. The bitterness of taste of the formulation diminishes due to increase in the ratio of the $\beta$-form of GM, which has poor wettability. The objective of this study was to find the optimum conditions for rapid formation of $\beta$-form of GM during the manufacturing process of the formulation. For this purpose, we focused on the transformation of $\alpha$-form of GM to $\beta$-form under the congealing conditions.

\section{Experimental \\ Material Glyceryl monostearate of $\beta$-form (GM, Taiyo Kagaku Co., Ltd.) was of the grade specified in the Japanese Pharmaceutical Excipients} Directory.
Preparation of Polymorphs of GM In preparing the $\alpha$-form of GM, GM melted at $120^{\circ} \mathrm{C}$ was dropped on the rotating wing of a stirring machine with an approximately $5 \mathrm{~cm}$ blade at $1500 \mathrm{rpm}$. Dripped GM was micronized, solidified, and recovered on the bed under room temperature.

GM with various ratios of polymorphs $\alpha$ and $\beta$ was prepared by mixing the $\alpha$-form immediately after preparation and the commercially available $\mathrm{GM}$ ( $\beta$-form) at the ratios of $25: 75,50: 50$ and $75: 25$.

Method of Heat-Treatment About $2 \mathrm{mg}$ of GM was placed in the differential scanning calorimeter (DSC) pan and stored for fixed times and temperatures.

Measurement of Powder Characteristics Thermal analysis was carried out using a differential scanning calorimeter (DSC-7, Perkin Elmer). About $2 \mathrm{mg}$ of GM was heat-treated in the opened pan in the DSC and, after cooling the sample to $30^{\circ} \mathrm{C}$ at the rate of $10^{\circ} \mathrm{C} / \mathrm{min}$, the first run of thermal analysis was carried out at a heating rate of $10^{\circ} \mathrm{C} / \mathrm{min}$ in the temperature range of $30^{\circ} \mathrm{C}$ to $90^{\circ} \mathrm{C}$. After cooling, the second run of thermal analysis was carried out under the same conditions. Enthalpies of first and second thermal analyses were expressed by the enthalpy change, $\Delta H_{1}$ and $\Delta H_{2}$, respectively.

Powder XRD patterns were obtained using a Rigaku Geigerflex RAD powder X-ray diffractometer under the following conditions: target, $\mathrm{Cu}$; filter, nonuse; voltage, $40 \mathrm{kV}$; current, $30 \mathrm{~mA}$; scanning speed, $4 \% \mathrm{~min}$.

\section{Results and Discussion}

XRD and Thermal Analyses of GM of $\alpha$-Form, $\beta$-Form and Their Mixtures The powder XRD patterns of two kinds of GM are shown in Fig. 1. Based on their patterns, the commercially obtained GM of the grade specified in the Japanese Pharmaceutical Excipients Directory was the $\beta$ form, while the GM that had melted and solidified was $\alpha$ form. The DSC curves of the $\alpha$-form and $\beta$-form are shown in Fig. 2; they had endothermic peaks at $67.9^{\circ} \mathrm{C}$ and $71.9^{\circ} \mathrm{C}$, respectively.

The patterns of peaks in the first run of thermal analysis were compared with those in the second run, and the enthalpy in the first analysis was compared with that in the sec- 


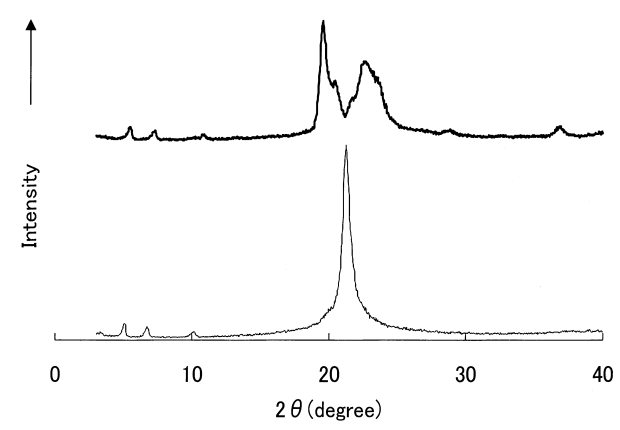

Fig. 1. Powder XRD Patterns of Two Kinds of Glyceryl Monostearate - GM of the grade specified in Japanese Pharmaceutical Excipients Directory ( $\beta$-form); $-\mathrm{GM}$ melted at $120^{\circ} \mathrm{C}$ and solidified $(\alpha$-form).

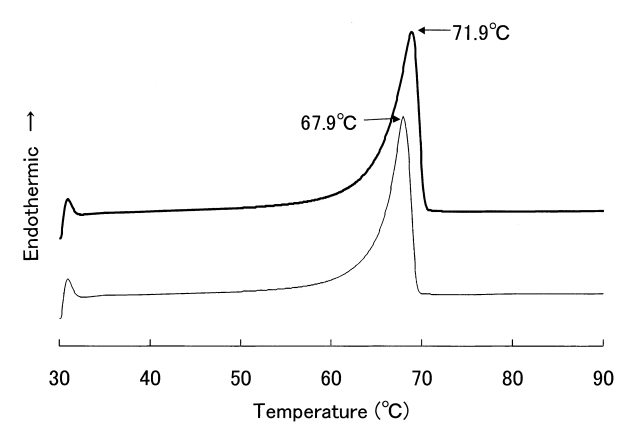

Fig. 2. DSC Curves for $\alpha$-Form and $\beta$-Form of Glyceryl Monostearate _ GM of the grade specified in Japanese Pharmaceutical Excipients Directory ( $\beta$-form);,$- \mathrm{GM}$ melted at $120^{\circ} \mathrm{C}$ and solidified $(\alpha$-form).

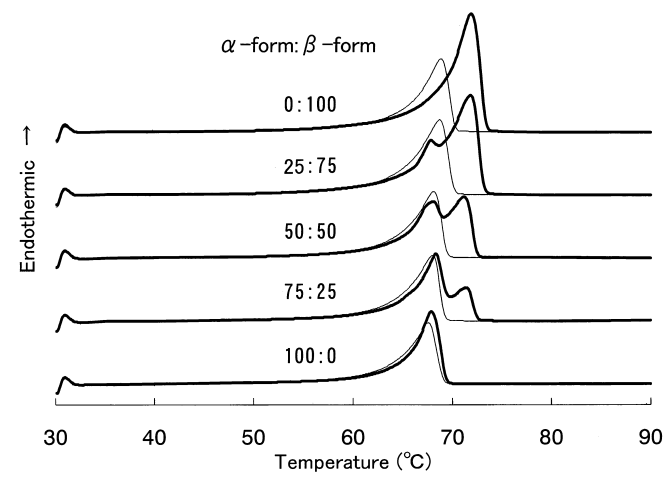

Fig. 3. DSC Curves for Physical Mixtures of $\alpha$-Form and $\beta$-Form at Various Ratios

— First thermal analysis; __ , second thermal analysis.

ond analysis. Figure 3 shows DSC curves of mixtures of the $\alpha$-form and $\beta$-form in various ratios. In the first thermal analysis of mixtures, two endothermic peaks shown by bold lines in Fig. 3 were observed. However, in the second analysis, no difference in thin lines was observed in either position or intensity of the peak or in the enthalpy, which almost coincided with that of the $\alpha$-form. Thus, the $\beta$-form in each mixture had been transformed to the $\alpha$-form during the first thermal analysis.

Determination of $\boldsymbol{\beta}$-Form Content in GM In Fig. 3, $\Delta H_{1}$ and $\Delta H_{2}$ are enthalpies calculated from the peak area of DSC between $40^{\circ} \mathrm{C}$ and $75^{\circ} \mathrm{C}$ in the first and second thermal analysis, respectively. $\Delta H_{1}$ was always larger than $\Delta H_{2}$, and $\Delta H_{1}$ increased and $\Delta H_{2}$ was constant, as the ratio of the $\beta$ form increased.

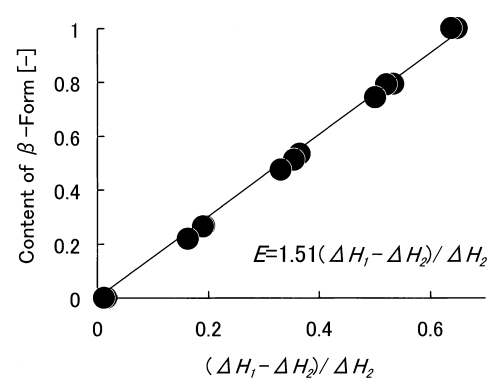

Fig. 4. Relationships between Enthalpy Change and $\beta$-Form Content

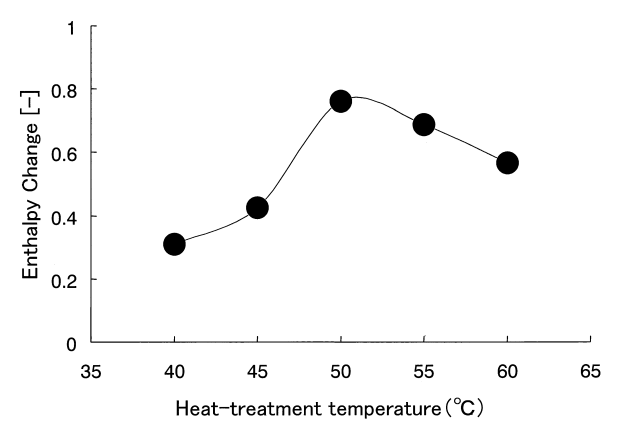

Fig. 5. Enthalpy Change of GM Heat-Treated at Each Temperature for $90 \mathrm{~min}$

Figure 4 shows the relationship between $\beta$-form content of mixture, $E$, and the enthalpy change, $\left(\Delta H_{1}-\Delta H_{2}\right) / \Delta H_{2}$. From the strong correlation observed, the following linear regression equation was obtained:

$$
E=1.51\left(\Delta H_{1}-\Delta H_{2}\right) / \Delta H_{2}
$$

where $E$ was the content of $\beta$-form in the mixture, and can be determined by repeating thermal analysis twice and obtaining $\Delta H_{1}$ and $\Delta H_{2}$.

Determination of Optimum Processing Temperature for Transformation $\alpha$-Form samples were heat-treated in the pan thermal analyzer at various temperatures and, after cooling, the enthalpy changes of GM were determined. The heat-treatment time was $90 \mathrm{~min}$, since this was the time that the maximum slope of enthalpy change was observed in our preliminary examination. The results are shown in Fig. 5.

Below $50{ }^{\circ} \mathrm{C}$, the enthalpy change increased as the heattreatment temperature increased. In contrast, it decreased as the temperature increased above $50^{\circ} \mathrm{C}$, since part of the GM probably melted and was transformed into $\alpha$-form by the heat-treatment. It is likely that the melted part would be glyceryl monomyristate with a low melting point. These results indicated that the optimum temperature in the heat-treatment for transformation was $50^{\circ} \mathrm{C}$.

Analysis of Transformation Rate Process during HeatTreatment The effect of the heat-treatment time in the DSC pan on the enthalpy change from the $\alpha$-form to the $\beta$ form was examined at $50^{\circ} \mathrm{C}$, and the results are shown in Fig. 6. Referring to the method of Hancock and Sharp, ${ }^{7)}$ the first-order equation was chosen for the transformation from the $\alpha$-form to the $\beta$-form of GM. Figure 7 shows the firstorder plot for the enthalpy change $(E)$ of $\mathrm{GM}$ treated at $50^{\circ} \mathrm{C}$ for each processing time. The transformation profile exhibited a two-phase pattern with initial quick ascent and subsequent slow increase. A point of inflection exists at about $3 \mathrm{~h}$. 


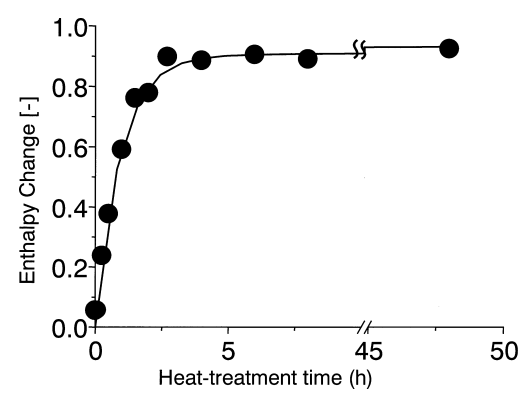

Fig. 6. Enthalpy Change of GM Treated at $50^{\circ} \mathrm{C}$ for Each Heat-Treatment Time

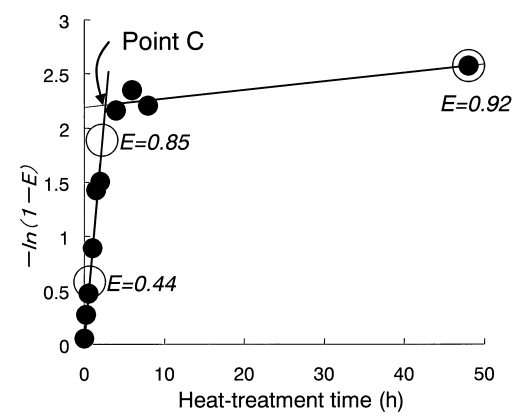

Fig. 7. First-Order Plot for Enthalpy Change $(E)$ of GM Treated at $50^{\circ} \mathrm{C}$ for Each Heat-Treatment Time

The intersection of the two lines in Fig. 7 was defined as Point C.

Figure 8 shows the effect of the heat-treatment time at $50{ }^{\circ} \mathrm{C}$ on the endothermic peak point. The peak profile exhibited a two-phase pattern in Fig. 7. A point of inflection exists at about $2 \mathrm{~h}$.

The X-ray diffraction patterns for $E=0.44,0.85$, and 0.92 shown in Fig. 7 are shown in Fig. 9. When $E$ was 0.44 , specific peaks of the $\alpha$-form and the $\beta$-form were observed in the X-ray diffraction pattern, while when $E$ was more than 0.85 , only the peak of the $\beta$-form shown in Fig. 1 was observed.

Maruyama et al. $^{2,3)}$ reported that the $\alpha$-form was transformed to the $\beta$-form via $\beta^{\prime}$-form above $25^{\circ} \mathrm{C}$; however, the point transformed from $\alpha$-form to $\beta^{\prime}$-form could not be detected with the X-ray diffraction method. We therefore assumed that the $\alpha$-form transforms to $\beta^{\prime}$-form at Point $\mathrm{C}$, which is the intersection of the two lines in Fig. 7. At Point $\mathrm{C}$, all of the $\alpha$-form transforms to $\beta^{\prime}$-form, and thereafter only transformation from $\beta^{\prime}$-form to the $\beta$-form occurs. The rate of transformation of GM crystals from $\alpha$-form to $\beta$-form can therefore be written as a consecutive reaction as follows.

$$
\alpha \stackrel{k_{1}}{\longrightarrow} \beta^{\prime} \stackrel{k_{2}}{\longrightarrow} \beta
$$

The rate equations are written as follows.

$$
\begin{aligned}
& Y_{\alpha}=\exp \left(-k_{1} \cdot t\right) \\
& Y_{\beta^{\prime}}=k_{1} /\left(k_{2}-k_{1}\right) \cdot\left(\exp \left(-k_{1} \cdot t\right)-\exp \left(-k_{2} \cdot t\right)\right) \\
& Y_{\beta}=1+1 /\left(k_{1}-k_{2}\right) \cdot\left(k_{2} \cdot \exp \left(-k_{1} \cdot t\right)-k_{1} \cdot \exp \left(-k_{2} \cdot t\right)\right)
\end{aligned}
$$

where $Y_{\alpha}, Y_{\beta^{\prime}}$, and $Y_{\beta}$ denote the percentages of the $\alpha$-form, $\beta^{\prime}$-form, and $\beta$-form present at each time $(t) . k_{1}$ and $k_{2}$ de-

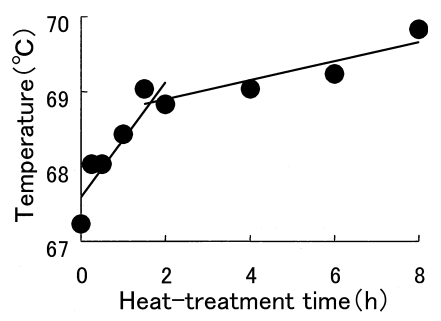

Fig. 8. Effect of Heat-Treatment Time at $50{ }^{\circ} \mathrm{C}$ on Endothermic Peak Point

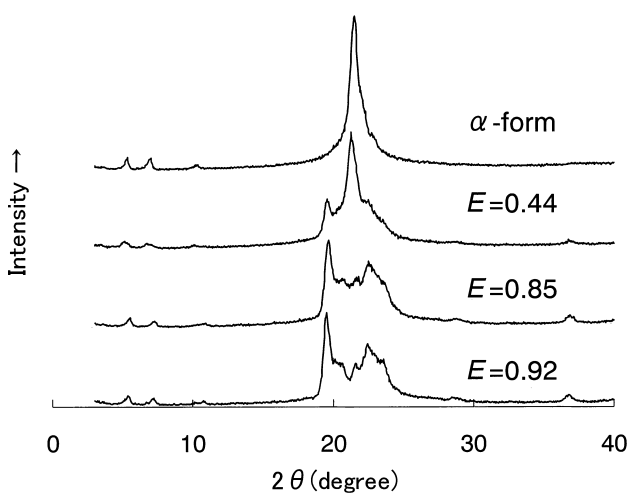

Fig. 9. X-Ray Diffraction Patterns for $E=0.44,0.85$, and 0.92 The heat-treatment time required for each $E$ value at $50{ }^{\circ} \mathrm{C}$ is shown in Fig. 7.

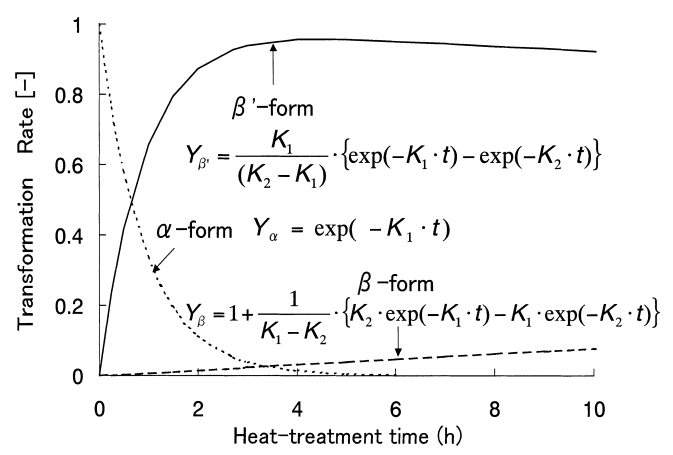

Fig. 10. Simulated Transformations of $\alpha$-, $\beta^{\prime}$-, and $\beta$-Forms

note the rates of transformation from $\alpha$-form to $\beta^{\prime}$-form and from $\beta^{\prime}$-form to the $\beta$-form, respectively.

The heat-treatment time at Point $\mathrm{C}$ was denoted as $t_{\mathrm{c}}$. Furthermore, the enthalpy change can be expressed by Eq. (5)

$$
E(t)=E\left(t_{\mathrm{c}}\right) \cdot\left(1-Y_{\alpha}\right)+\left(1-E\left(t_{\mathrm{c}}\right)\right) \cdot Y_{\beta}
$$

where $E\left(t_{\mathrm{c}}\right)$ denotes the enthalpy change at $t_{\mathrm{c}} . E\left(t_{\mathrm{c}}\right), k_{1}$, and $k_{2}$ were calculated by the nonlinear least-squares method and were determined to be $0.902,1.074$, and 0.0074 , respectively.

Values of $Y_{\alpha}, Y_{\beta^{\prime}}$, and $Y_{\beta}$ are shown in Fig. 10, and at $2 \mathrm{~h}$, were about $0.11,0.88$ and 0.01 , respectively.

\section{Conclusion}

The enthalpy change $(E)$ of GM during the heat-treatment was determined by a series of thermal analyses in the DSC pan. Below $50^{\circ} \mathrm{C}, E$ increased as temperature increased, while it decreased as temperature increased above $50^{\circ} \mathrm{C}$. The effect of processing time in the pan on $E$ from the $\alpha$-form to 
the $\beta$-form was examined at $50^{\circ} \mathrm{C}$. The profile of $-\ln (1-E)$ exhibited a two-phase pattern with a point of refraction.

We assumed that all the $\alpha$-form transforms to $\beta^{\prime}$-form at a certain point (point $\mathrm{C}$ in Fig. 7), and subsequently that only transformation from $\beta^{\prime}$-form to the $\beta$-form occurs; these changes follow those of consecutive reactions. Based on this hypothesis, the transformation rate equations were deduced. The experimental data obtained coincided well with the theoretical curve drawn with the equations.

In conclusion, $50^{\circ} \mathrm{C}$ was found to be the optimum processing temperature for the transformation of GM from $\alpha$ form to $\beta$-form.

\section{References and Notes}

1) A portion of this work was presented at the 16th Annual Meeting of the Academy of Pharmaceutical Science and Technology, Japan, March 2001.

2) Maruyama T., Niiya I., Imamura M., Okada M., Matsumoto T., Horisawa M., Mastumoto T., Yukagaku, 20, 395-402 (1971).

3) Maruyama T., Niiya I., Imamura M., Okada M., Matsumoto T., Yukagaku, 22, 85-88 (1973).

4) Hidaka T., Yukagaku, 30, 823-836 (1981).

5) Yajima T., Nogata A., Demachi M., Umeki N., Itai S., Yunoki N., Nemoto M., Chem. Pharm. Bull., 44, 187-191 (1996).

6) Yajima T., Umeki N., Itai S., Chem. Pharm. Bull., 47, 220-225 (1999).

7) Hancock J. E., Sharp K. H., J. Am. Ceram. Soc., 55, 74-77 (1972). 